EYECAD 360, инструменту, который позволяет создавать несколько визуализаций фотосферы, которые можно просматривать как со смартфона и ПК.

Calvin

Программа Calvin (Collaborative Architectural Layout Via Immersive Navigation), применяться для проверки возможностей виртуальной реальности в проектировании сооружений и в совместной работе с визуальными объектами. В данном приложении виртуальная реальность служит не только для визуализации проектов, но и для создания многочисленных перспективных планов.

Таким образом, на данном этапе информационной индустрии, получены возможности виртуальной реальности в проектировании зданий и в совместной работе с визуальными объектами. Архитекторы получили программные средства, которые позволяют им применять при проектировании технологию виртуальной реальности.

***

1. Harris, Laurence R.; Jenkin, Michael R. M. (2011). Vision in 3D Environments. Cambridge, UK: Cambridge University Press. p. 311.

2. Ervin, Garip; Banu, Garip, S. (2021). Handbook of Research on Methodologies for Design and Production Practices in Interior Architecture. Hershey, PA: IGI Global. p. 396.

3. Kharchenko L., Panova I., Babaeva E., Magomedova R., Gulyakin D., Pashtaev B. Russian syndromes, impeding the development of the innovation processes in education // Life Sci J 2014; 11 (9s): 184 -187, USA, (ISSN: 1097-8135). http://www.lifesciencesite.com. 35.

4. Гулякин Д.В. Современный вуз как среда, реплицирующая культуру общества // Научное обозрение. Серия 2: Гуманитарные науки. - 2012. - № 6. - С. 91-95.

5. Gisi, M. A., and Sacchi, C. "Co-CAD: A Collaborative Mechanical CAD System”, PRESENCE, MIT Press, Vol. 3, No. 4, Fall 1994, pp. 341-350.

6. Greenberg, S., and Marwood, D. "Real Time Groupware as a Distributed System: Concurrency Control and its Effect on the Interface", CSCW 94, ACM, 1994, pp. 207-217.

\title{
Панченко Н.М. \\ К вопросу оценки качества функционирования технологических процессов в строительстве через их производительность и сроки выполнения работ
}

Петербургский государственный университет путей сообщения Императора Александра I doi: 10.18411/trnio-10-2021-64

(Россия, Санкт-Петербург)

\section{Аннотация}

Понятие «качество» является философской категорией, оно сложно и многогранно. Множество работ российских и зарубежных учёных посвящено проблемам качества выпускаемой продукции, оказываемых услуг, жизненного цикла продукции и т.д. В данной статье затрагиваются вопросы оценки качества строительной продукции и функционирования производственных процессов. Качество строительной продукции зависит от качества функционирования производственного процесса в результате которого она создаётся и закладывается ещё на стадии проектирования этого процесса. Качество является относительной величиной и оценить его в абсолютных единицах невозможно. Но для качественного сравнения функционирования технологических процессов возможно использовать такие показатели, как производительность процесса, сроки выполнения работ, себестоимость, механовооруженность и др. Все эти показатели отображают надёжность функционирования производственного процесса, взаимосвязаны между собой и могут служить показателями качества функционирования технологических процессов в строительстве.

Ключевые слова: качество, показатели качества, производительность, надёжность, большая система, технология, строительный процесс, себестоимость.

\section{Abstract}

The concept of "quality" is a complex and multifaceted philosophical category. A lot of researches of Russian and foreign scientists are devoted to the problems of the quality of products, 
services provided, the life cycle of products, etc. This article deals with the issues of assessing the quality of construction products and production processes. The quality of construction products depends on the quality of the production process and is a result of the design stage. Quality is a relative quantity and it is impossible to evaluate it in absolute units, but for a comparison of the technological processes functioning, it is possible to use such indicators as: process productivity, work completion time, cost, mechanical strength, etc. All these indicators reflect the reliability of the production process and are interconnected with each other.

Keywords: quality, quality indicators, productivity, reliability, large system, technology, construction process, cost price.

Качество - это сложное комплексное понятие, которое опирается на ряд таких понятий, как: «свойство», «упорядоченность», «структура», «количество», «система», «устойчивость», «целостность», надёжность» и другие. Предметом исследования в теории качества являются следующие категории:

- факторы формирования качества;

- у устойчивость показателей качества в процессе эксплуатации;

- измерение и оценка качества;

- н надёжность элементов составляющих большую систему и др.

Теория качества рассматривает категорию «качество» в различных аспектах: технических, экономических, социальных и в их единстве [1]. Развитию теории качества посвящены работы таких отечественных учёных, как Атаев С.С., Бусленко Н.П., Гусаков А.А., Монфред Ю.Б., Неснов В.И. и др. Большинство работ в данной области затрагивают вопросы исследования свойств качества продукции потребления и лишь отдельные из них качеству строительного производства [2], [3], [4].

Качество продукции строительного производства напрямую зависит от качества функционирования технологического строительного процесса. Под качеством функционирования технологического строительного процесса будем понимать совокупность свойств этого процесса, благодаря которым, возникает возможность создания строительной продукции с заданной надёжностью, в необходимом количестве и в соответствии с уровнем научно-технического прогресса (НТП). Основы качества функционирования технологического строительного процесса должны быть заложены на стадии проектирования и поддерживаться в процессе непосредственного функционирования строительного процесса. Именно на стадии проектирования определяется технологичность процессов, которая является основным фактором, влияющим на качество и количество выпускаемой продукции [5].

Технология, применяемая в процессе строительства, оказывает серьёзное влияние на количественный состав рабочих и единиц техники занятых в данном процессе, на квалификацию рабочих и расположение техники, а следовательно, и на структуру трудозатрат, приходящихся на единицу готовой продукции. Технологию производства работ необходимо выбирать исходя из типа выпускаемой строительной продукции и в соответствии с уровнем НТП для того, чтобы обеспечить выпуск продукции заданного качества. Это достигается путём правильного выбора эксплуатационных характеристик строительных машин и схем структурно-технологической компоновки производственного процесса.

Понятие «качество» для любой продукции или процесса имеет определённые границы, за пределами которого уже существует «качество» иного рода, то есть любые показатели качества могут быть измерены в пределах неких границ, то есть иметь количественную оценку [4]. Для того, чтобы оценить качество функционирования строительных процессов с количественной стороны, необходимо обратиться к таким признакам функционирования строительных процессов, как: соответствие применяемой технологии уровню развития используемой в ней техники, надёжность и долговечность процесса, количество и уровень квалификации занятых в процессе рабочих и др. Качество - 
это относительная величина, поэтому и оценить её в абсолютных единицах невозможно. Но для качественного сравнения технологий функционирования строительных процессов возможно использовать такие показатели, как: производительность, сроки выполнения работ, себестоимость, механовооруженность и др. [6].

Под производительностью строительного процесса следует понимать количество доброкачественной продукции, создаваемой им при функционировании в единицу времени. Производительность строительного процесса зависит главным образом от уровня технического развития отрасли, которым в свою очередь, определяется выбор технологий и технических средств, применяемых в процессе строительства. Иными словами, производительность строительного процесса зависит от качества применяемых технологий. В оценке качества применяемых технологий производительность процесса является сравнительной величиной, которая характеризует качество в численных натуральных показателях. Все остальные показатели качества функционирования строительных процессов характеризуют его опосредованно, через производительность. Поэтому производительность процесса можно считать одним из основных показателей качества его функционирования.

Технология строительного процесса определяет его структуру и соответствие уровню развития техники, а надёжность процесса определяет совместное действие технологии и техники [7]. Любой строительный процесс состоит из определённого конечного числа операций, которые соединены между собой в определённой последовательности. Элементы человеко-технологических систем (ЧТС) могут иметь между собой последовательное, параллельное или смешанное соединения. Благодаря этому любой технологический процесс в области строительства следует рассматривать, как сложную большую систему, элементы которой работают, отказывают и восстанавливаются по-своему. Поэтому надёжность процессов отображает одновременное действие множества факторов и является показателем качества функционирования строительных процессов. Надёжность функционирования строительных процессов напрямую связана с их производительностью. Для исследования специфики оценки надёжности функционирования строительных процессов необходимо использовать математический аппарат теории решения больших систем, который достаточно хорошо разработан отечественными и зарубежными учёными [3].

Сроки производства работ и себестоимость произведённой строительной продукции напрямую связаны с производительностью функционирующего процесса и зависят от изменения производительности данного процесса. При снижении производительности как результат получаем увеличение сроков производства работ и себестоимости строительной продукции, при повышении производительности, наоборот, сокращение сроков производства работ и снижение себестоимости строительной продукции [8]. Поэтому, срок производства работ и себестоимость строительной продукции, как и производительность процесса, являются показателями качества функционирования технологических процессов в строительстве.

$$
* * *
$$

1. Неснов В.И. Бизнес и политика в теории эргатических систем. СПб. 2000. 142с.

2. Гусаков А.А. Системотехника строительства. М., Стройиздат. 1993. 440с.

3. Гусаков А.А., Монфред Ю.Б., Прыкин Б.В. Моделирование организационно-технологической надёжности строительства. М., SVR-Аргус, 1994. 472 с.

4. Губинский А.И. Надёжность и качество функционирования эргатических систем. Л., Наука. 1982. $262 \mathrm{c}$.

5. Рогонский В. А., Воронин В. М. Строительные катастрофы СПб.: Стройиздат СПб., 2001. - 157 с.

6. Панченко Н.М. Методологические основы комплексного проектирования сложных строительных процессов (на примере производства земляных работ). Автореферат диссертации. СПб., 1999. 25 с.

7. Панченко Н.М. К вопросу выбора комплекта строительной техники при производстве земляных работ. Вопросы образования и науки. Сборник научных трудов по материалам международной научнопрактической конференции 30 июня 2020 г. Часть 1. Тамбов 2020. Стр. 85-87

8. Панченко Н.М. К проблеме надёжности строительных процессов и путям её решения. Наука, образование, общество. Сборник научных трудов по материалам международной научно-практической конференции 29 февраля 2016 г. Часть 2 Тамбов 2016. Стр. 86-88 